\title{
Metapopulations revisited: the area-dependence of dispersal matters
}

\author{
Shaopeng Wang (iD) ${ }^{1}$ and Florian Altermatt (iD) $2,3,4$ \\ ${ }^{1}$ Key Laboratory for Earth Surface Processes of the Ministry of Education, Institute of Ecology, College of Urban and Environmental \\ Sciences, Peking University, Beijing 100871 China \\ ${ }^{2}$ Department of Evolutionary Biology and Environmental Studies, University of Zurich, Winterthurerstrasse 190, Zürich CH-8057 \\ Switzerland \\ ${ }^{3}$ Department of Aquatic Ecology, Eawag, Swiss Federal Institute of Aquatic Science and Technology, Überlandstrasse 133, Dübendorf \\ CH-8600 Switzerland
}

Citation: Wang, S., and F. Altermatt. 2019. Metapopulations revisited: the area-dependence of dispersal matters. Ecology 100(9):e02792. 10.1002/ecy.2792

\begin{abstract}
The metapopulation concept initiated a paradigm shift in ecology and conservation biology, recognizing the eminent role of dispersal and colonization as fundamental processes contributing to species' long-term persistence. Early models made ad hoc assumptions about a positive area dependency of dispersal (i.e., total number of emigrants), which persisted in the theoretical literature; however, numerous empirical examples of negative area dependencies of dispersal have been reported. Here, we first give a qualitative overview for different area dependencies of dispersal in empirical systems. Then, using a spatially realistic Levins model, we show that extending assumptions on the area dependence of dispersal (ADD) to include all empirically supported parameter space, specifically also negative ADD, alters predictions on several conservation-relevant patterns. Importantly, we find that small patches could be of similar importance as large ones if dispersal decreases inversely with patch area, a result contrasting with previous findings based on a positive ADD. This leads to context-dependent strategies to preserve metapopulations. If dispersal is positively correlated with patch area, efforts should be devoted to preserving large patches and the total habitat area. If dispersal is negatively correlated with patch area, the most efficient strategy is to preserve a high number of patches, including small ones. Our results have direct implications for management decisions in the context of destruction, deterioration, and protection of habitat patches.
\end{abstract}

Key words: colonization; dispersal rate; emigration; habitat destruction; metapopulation capacity; patch area; patch size; SLOSS.

\section{INTRODUCTION}

The metapopulation concept was a major breakthrough and paradigm shift in ecology (Gilpin and Hanski 1991, Hanski 1998, Hanski and Gaggiotti 2004). Before its development (Levins 1969, 1970), ecological models had mostly assumed that the dynamics and persistence of populations can be described by local factors and from a localized and equilibrium perspective (Levin 1992). The metapopulation concept showed how a species can still persist regionally while every local population is doomed and has a finite extinction risk.

As a consequence, the focus of theoretical ecology, and eventually also empirical ecology and conservation biology, shifted from a localized perspective to a spatial perspective (Clobert et al. 2001). Extensive consecutive work showed that dispersal and metapopulation dynamics are important for a wide range of settings and organisms (Gilpin and Hanski 1991, Hanski 1998, Hanski and Gaggiotti 2004), and the original models were expanded

Manuscript received 29 March 2019; accepted 28 May 2019. Corresponding Editor: José Miguel Ponciano.

${ }^{4}$ E-mail: Florian.Altermatt@eawag.ch in various aspects, including consideration of spatially explicit and realistic dispersal networks (e.g., Hanski 1999, Gilarranz and Bascompte 2012, Mari et al. 2014), stability of patches (e.g., Reigada et al. 2015, Wang et al. 2015), species interactions (e.g., Leibold et al. 2004, Fournier et al. 2017), evolution and genetics (e.g., Hanski and Saccheri 2006, Jansen and Vitalis 2007, Saastamoinen et al. 2018), and ecoevolutionary feedbacks (e.g., Hanski et al. 2011, Fronhofer and Altermatt 2017).

Earlier theoretical work assumed that all patches were identical, and either in an empty or occupied state (Levins 1969). Harrison (1991) was one of the first, clearly recognizing that patches in a metapopulation vary in quality and size, and that this variation may affect population sizes and subsequently metapopulation dynamics. One implication of an almost universal variation in patch size in natural metapopulations is a subsequent variation in the number (quantity) of dispersers per patch. Consequently, theoretical work started to relax the assumption that all habitat patches are identical and generally assumed that the number of dispersers produced per patch is an increasing function of both patch lifetime and patch area. This early-on "assumption" of a positive area dependence of dispersal (ADD) 
postulated and used in seminal theoretical work (Gyllenberg and Hanski 1992, 1997, Hanski and Gyllenberg 1993, Travis et al. 1999, Metz and Gyllenberg 2001, Poethke and Hovestadt 2002; but see Kindvall and Petersson 2000) subsequently immersed the empirical literature as an "expected," but rarely tested, relationship.

More recently, it was put forward that the dependence between patch size and dispersal/emigration needs to be looked at (e.g., Stamps et al. 1987, Englund and Hambäck 2004a, Hambäck and Englund 2005, Altermatt and Ebert 2010), and individual empirical studies started to reveal that the area dependence of dispersal is not as unequivocal as one would expect. It was found that dispersal, or the total number of emigrants, is not only positively related, but can also be negatively related, to patch size. For example, high numbers of emigrants from small patches were found in a vole metapopulation (Crone et al. 2001), in phytophagous beetles. and some lepidoptera (e.g., Turchin 1986, Hjermann 2000, Menéndez et al. 2002). Similarly, in ephemeral rock pools with frequent desiccation and subsequent exposition of migration stages to dispersal, it was found that most dispersers at the metapopulation level originated from small pools (Altermatt et al. 2008, 2009, Altermatt and Ebert 2010). The observation of negative ADD in natural metapopulations obviously requires also some evolutionary explanation. Although not the focus of our paper, the study of dispersal evolution in metapopulations has a long tradition (e.g., Olivieri et al. 1995, Travis and Dytham 1998, Ronce 2007, Fronhofer and Altermatt 2017). From an evolutionary perspective, a negative ADD can emerge under certain conditions, for example, when there is strong, patch-size-dependent Allee effects. In this case, individuals have problems finding mates in small patches, so high levels of dispersal are expected to evolve. This scenario may be realistic for a well-studied metapopulation of voles (Pokki 1981, Crone et al. 2001). Another, mutually nonexclusive scenario resulting in the evolution of high levels of dispersal in small patches is expected in cases where patch area and patch stability are correlated. For example, when small patches are more (or more often) affected by perturbations or are more ephemeral, environmental change cannot only induce dormancy, but would likely also evolve to trigger high levels of dispersal, especially in small patches. The dependency of dispersal on patch stability and/or patch disturbances is relatively well documented and also seems to have a genetic basis in natural metapopulations (Purves and Dushoff 2005, Vanschoenwinkel et al. 2008, Berendonk et al. 2009, Reigada et al. 2015). Despite these varying dependencies of dispersal on patch area (from positive to negative; see also our literature survey below) theoretical models continued to consider mostly a positive ADD. Integrating all realized parameter spaces of ADD into metapopulation models is important because we expect negative ADD to lead to qualitatively different predictions than those claimed by current theory using positive ADD.
A quantitative framework to understand the effects of patch area and locations on the persistence of metapopulations was developed by Hanski and Ovaskainen (2000), who considered mainly a positive dependence of the overall dispersal on patch area (i.e., positive ADD), particularly a linear or power-law increase of dispersal with area (Ovaskainen and Hanski 2001, 2003). One prediction from these models is that larger patches are more important than smaller ones in the persistence of metapopulations (Hanski and Ovaskainen 2000), but these conclusions might not apply to observed natural metapopulations that exhibit opposite scenarios of area dependence of dispersal (e.g., Kareiva 1985, Turchin 1986, Crone et al. 2001, Bates et al. 2006, Altermatt and Ebert 2010).

In this paper, we first compiled empirical evidence for positive, neutral, and negative ADD. We then revisited metapopulation theory, and addressed the relevance of small patches on the overall metapopulation dynamics. Our model is built upon the upgrade of Levins' model by Hanski and Ovaskainen (2000), allowing for variation in patch size. We combine analytic and numerical approaches to investigate how assumptions on ADD alter the predictions on (1) the relationship between the importance of a patch for metapopulation persistence with its area, (2) the effects of area distribution of patches on the persistence of metapopulations, and (3) the consequences of habitat destruction and deterioration. We note that our focus is on the dependence of total number of dispersers or emigrants on patch area, as this is directly compatible with the assumptions in the classical metapopulation models (Hanski and Ovaskainen 2000, Ovaskainen and Hanski 2001, Ovaskainen 2003). This differs from approaches looking at the density dependence of dispersal or the area dependence of dispersal rates (i.e., dispersal propensity per individual), both of which are also commonly found in the literature (Matthysen 2005, Støen et al. 2006). For instance, many empirical studies have revealed that dispersal rates not only depended on the density of the focal species, but also on the density of its competitors, prey, and predators (Matthysen 2005, De Roissart et al. 2015, Fronhofer et al. 2015, 2018). Although clearly interesting, we will not elaborate further on those aspects, but focus instead on the area dependence of dispersal on metapopulation dynamics and stability. This has rarely been studied, but is critically needed to apply metapopulation models in a conservation perspective realistically.

\section{Methods}

The framework for studying area dependence of dispersal $(A D D)$

To investigate the effect of ADD, we consider a spatially realistic (i.e., having variation in patch size and distance between patches) version of the Levins model 
(Ovaskainen 2003), in which the dynamics of the probability of patch $i$ being occupied $\left(p_{i}\right)$ is:

$$
\frac{d p_{i}}{d t}=c \cdot \sum_{j \neq i} \exp \left(-\alpha d_{i j}\right) A_{j}^{\omega} p_{j} \cdot\left(1-p_{i}\right)-\frac{e}{A_{i}} \cdot p_{i}
$$

where $A_{i}$ is the area of patch $i, d_{i j}$ is the distance between patch $i$ and $j$, and $c$ and $e$ are constants. The first term on the right-hand side of Eq. 1 captures the rate of recolonization of an empty patch, as realized by dispersers from other patches. $\alpha$ is a parameter that captures the scale of dispersal (i.e., $1 / \alpha$ is the characteristic length). The parameter $\omega$ captures different scenarios of ADD (Fig. 1). When $\omega=1$, dispersal (i.e., total emigration) increases linearly with patch area, a scenario that was most often considered in previous models (Hanski and Ovaskainen 2000, Ovaskainen and Hanski 2001); when $\omega=-1$, dispersal decreases reciprocally with patch area, a scenario observed in some empirical studies (Altermatt and Ebert 2008, 2010) but rarely explored in previous models; when $\omega=0$, dispersal is independent of patch area, which is an intermediate scenario between the above two. The second term in Eq. 1 captures the extinction rate of an occupied patch, which is assumed to be inversely related with patch area. We also examine an alternative model assuming an exponential decay of extinction rate with patch area, which generates qualitatively similar results (Appendix S2: Fig. S1).

Hanski and Ovaskainen had analyzed models that are close variants of Eq. 1 in detail (Hanski and Ovaskainen 2000, Ovaskainen and Hanski 2001). One remarkable result from their work is that the condition for the metapopulation to persist on the landscape is: $\lambda>e / c$. Here $\lambda$ is referred to as the metapopulation capacity and is calculated by the dominant eigenvalue of the landscape matrix $M$, the elements of which are

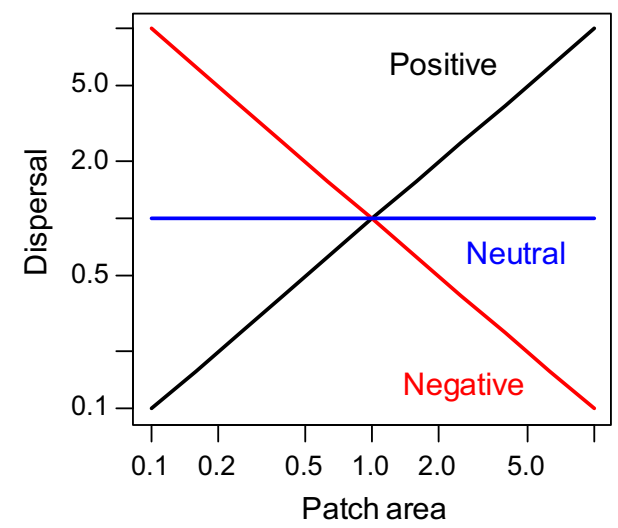

FIG. 1. Different scenarios of the area dependence of dispersal (ADD). When $\omega=1$, dispersal (emigration) increases linearly with patch area (black line). When $\omega=-1$, dispersal decreases reciprocally with patch area (red line). We also consider an independent or neutral case, i.e., $\omega=0$ (blue line). Note that $x$ - and $y$-axes are both on logarithmic scales.

$$
m_{i j}=\left\{\begin{array}{ll}
0 & i=j \\
\exp \left(-\alpha d_{i j}\right) \cdot A_{i} \cdot A_{j}^{\omega} & i \neq j
\end{array} .\right.
$$

The concept of metapopulation capacity thus provides a quantitative tool to assess the effects of habitat area and locations on the persistence of a metapopulation living in a patchy landscape.

\section{Overview of empirical work on dispersal in metapopulations}

In order to get a better understanding of the relationships between patch area and dispersal (emigration), we screened the literature for ADD. We did not aim for a quantitative review, but rather wanted to get a qualitative overview of the types of area dependence of dispersal observed to justify the parameter space explored with our model. In a first step, we did two Web of Science searches on 2 February 2019, searching for "patch area AND metapopulation AND emigration" and "patch size AND emigration," respectively. We screened abstracts of 42 and 137 papers in total, respectively, and were interested in papers giving raw data to calculate ADD. In a second step, we complemented this search with crossreferenced papers and papers mentioned by Englund and Hambäck (2004a). We were interested in papers giving absolute numbers of dispersers (or where this could be calculated from) and actual patch area. We compiled 18 papers, ending up with a total 23 independent population-specific estimates of empirically observed ADD (Batch 1984, Kareiva 1985, Turchin 1986, Sutcliffe et al. 1997, Baguette et al. 2000, Hjermann 2000, Roland et al. 2000, Andreassen and Ims 2001, Bergman and Landin 2001, Crone et al. 2001, Menéndez et al. 2002, Välimäki and Itämies 2003, Englund and Hambäck 2004b, Wang et al. 2004, Bates et al. 2006, Altermatt and Ebert 2008, Fred and Brommer 2009, Andersson and Hambäck 2012). We note that this list is not comprehensive. Many additional studies also reported a relationship between patch area and per capita dispersal rates, including many negative relationships (e.g., Hill et al. 1996, Kindvall 1999, Wahlberg et al. 2002, Schtickzelle and Baguette 2003).

The selected studies cover many animal groups, such as aquatic invertebrates, butterflies, moths, beetles, crickets, and small mammals, and include both experimental as well as comparative studies. For each of these studies, we extracted (if possible) the raw values of all patch areas (in $\mathrm{m}^{2}$, except for Altermatt and Ebert 2008 for which patch size was described by volume) and the absolute number of dispersers for each of the populations studied. For 19 of the 23 populations, we then fitted a linear regression model between $\log$ (number of dispersers) $\sim \log$ (patch area). We used the predicted relationship for the range of patch areas covered by the respective studies. For two further metapopulations (Andersson and Hambäck 2012) only the mean values for three different 
patch area classes were given. For those, we directly used the interpolation between these three mean values. Finally, for two further metapopulations (Crone et al. 2001, Altermatt and Ebert 2008), the relationship between patch area and number of dispersers was unimodal and the respective dependency was extracted from the figures in the two papers (for the latter paper, the number of dispersal stages produced was multiplied by the likelihood of patch-specific dispersal defined by pool desiccation, such that it was directly comparable as effective number of dispersers). We plotted all of these empirically observed relationships between patch area and number of dispersers, and also summarized the slope estimates of all linear models predicting the relationship between patch area and dispersal in a histogram.

\section{Analysis of the model}

We combine analytic and numerical approaches to investigate how the assumption of ADD alters the predictions on the importance of individual patches, patch size distribution, and habitat destruction on the persistence of metapopulations. Our analytic approach builds on previous results by Hanski and Ovaskainen (Hanski and Ovaskainen 2000, Ovaskainen and Hanski 2001, Ovaskainen 2003), but we derive new solutions (e.g., Eqs. 2,3). Our numerical approach also extends the analytic results to general cases with spatially realistic landscape structure.

Patch importance.-Following Ovaskainen (2003), we defined the importance of a patch as its relative contribution to the metapopulation capacity: $I_{k}=\frac{\lambda-\lambda^{(-k)}}{\lambda}$, with $\lambda^{(-\mathrm{k})}$ denoting the metapopulation capacity if the patch $k$ is removed. One mathematical result derived by Ovaskainen (2003) is that the importance of patch $k$ can be approximated by: $I_{k} \approx \frac{y_{k} x_{k}}{\vec{v}}$, where $\vec{y}$ and $\vec{x}$ are the left and right leading eigenvectors of matrix $M$, respectively, and $y_{k}$ and $x_{k}$ are the $k$ th components of the respective vector. Based on this result, we derive a rough approximation for the relationship between patch importance and area (Appendix S1):

$$
I_{k} \propto A_{k}^{1+\omega}
$$

When $\omega=1$, we have $I_{k} \propto A_{k}^{2}$, as shown in Hanski and Ovaskainen (2000); when $\omega=0$, we have $I_{k} \propto A_{k}$; when $\omega=-1, I_{k}$ is independent of $A_{k}$. Thus, on a $\log -$ $\log$ scale, $I_{k}$ and $A_{k}$ are expected to exhibit a linear relationship with slopes of $1+\omega$, that is, 2 under positive ADD $(\omega=1), 1$ under neutral ADD $(\omega=0)$, and 0 under negative $\operatorname{ADD}(\omega=-1)$.

We then derive the importance of each patch numerically by simulating the loss of a particular patch and monitoring the change of metapopulation capacity. Under different scenarios of ADD, we examine the relationship between patch importance and area. We conduct regression analyses and derive the slope between patch importance and area on a log-log scale and compare these numerical slopes to analytic approximations given by Eq. 2 .

Effects of size distribution of patches.-We investigate, given the total area of habitats, the effect of size distribution of patches (i.e., patch number and size variation of patches) on metapopulation capacity under different scenarios of ADD. First, we examine how the number of patches may affect the metapopulation capacity. In a homogeneous landscape, that is, all patches have the same area and colonization rates, Ovaskainen (2002) showed that metapopulation capacity changes with the number of patches $(n)$ as: $\lambda \propto n^{-\omega}$. To extend their results to heterogeneous landscapes where habitat patches differ in their area and distances to each other, we perform simulations and examined the relationship between metapopulation capacity and number of patches under different values of ADD (i.e., $\omega=-1,-0.9, \ldots, 0.9,1)$. Second, given the total area and number of patches, the variation in patch size may also affect the metapopulation capacity. To investigate this, under different values of ADD, we simulate 100 metapopulations with the same total area and number of patches but with varying variances in patch size distribution. This was realized by first randomly sampling patch areas from log-uniform distributions with different variance and then normalizing them by a constant total area. We then investigate the relationship between the standard deviation of patch areas and metapopulation capacity.

Consequence of habitat destruction.-We investigate how metapopulation capacity changes following habitat destruction. We first consider habitat deterioration, where the number of patches remains the same but patch area decreases. In a special case in which all patches change in a coherent manner, that is, the area of all patches reduces to a proportion $\theta$ of their origins, the landscape matrix $M_{\theta}$ becomes

$$
m_{i j}(\theta)=\left\{\begin{array}{cc}
0 & i=j \\
\exp \left(-\alpha d_{i j}\right) \cdot\left(\theta A_{i}\right) \cdot\left(\theta A_{j}\right)^{\omega} & i \neq j
\end{array} .\right.
$$

It is easy to see that the metapopulation capacity following habitat deterioration is

$$
\lambda_{\theta}=\lambda \cdot \theta^{1+\omega}
$$

Such a scenario may happen when the number of patches stays constant, but their effective size changes, for example when ponds are only filled partially with water, or when desiccation makes moss patches effectively smaller (Hanski and Gaggiotti 2004). It predicts that, the metapopulation capacity decreases with habitat deterioration under positive $(\omega=1)$ and neutral $(\omega=0)$ ADD scenarios, and it does not change under the negative $\operatorname{ADD}(\omega=-1)$ 
scenario. In more general cases, we perform simulations by reducing the area of selected patches to a pre-assigned minimum area (so as to prevent the complete loss of the patch and keep the number of patches constant) and ensuring the total reduction of habitat area to match the pre-defined degree of habitat deterioration. We considered the three following scenarios: (1) large patches lost first, (2) small patches lost first, and (3) random loss.

We then consider habitat loss, where a number of patches become completely unhabitable. We simulate subsequent loss of habitat patches and monitor the changes in metapopulation capacity under different scenarios of ADD. Again, the loss of patches follows each of the three regimes: (1) large patches lost first, (2) small patches lost first, and (3) random loss. For a given proportion $(p)$ of remaining patches, we calculate the corresponding metapopulation capacity $\left(\lambda_{p}\right)$ and define the relative metapopulation capacity as $\lambda_{\text {rel }}(p)=\lambda_{p} / \lambda$. Under each distribution of patch areas and each regime of patch loss, we simulate 100 metapopulations and examine the patterns of $\lambda_{\text {rel }}(p)$ as a function of $p$.

\section{RESULTS}

Our analysis of empirical studies shows that positive, negative, neutral, and unimodal relationships between patch area and number of dispersers have been found across a wide range of animal groups, including aquatic and terrestrial invertebrates and small mammals (Fig. 2a). We also find that the distribution of slopes of all linear models (log-log scale) between patch area and number of dispersers covers the parameter space from 1 to 1 (Fig. 2b).

Our simulations show that within a metapopulation, the importance of a patch increases with its area when $\omega=1$ or 0 and has no relation when $\omega=-1$ (Fig. 3a). Across 100 simulated metapopulations, the median loglog slopes (and their 90\% confident interval) of regressions between patch importance and area are 1.99 (CI: [1.86, 2.13] when $\omega=1), 0.99$ (CI: [0.90, 1.12] when $\omega=0$ ), and -0.0003 (CI: $[-0.090,0.063]$ when $\omega=-1$ ), which are matching the analytic expectations of 2,1 , and 0 (Eq. 2; Fig. 3b). The qualitative results that a negative ADD always increases the relative importance of small patches and weakens that of large patches are robust to assumptions of an exponential decay of extinction rate with patch area, although the regression slopes change quantitatively and deviate from the expectations by Eq. 2 (Appendix S2).

Our simulations show that under different scenarios of ADD, the metapopulation capacity $\lambda$ is differently affected by the size distribution of patches. Given the total area of patches, $\lambda$ decreases with the number of
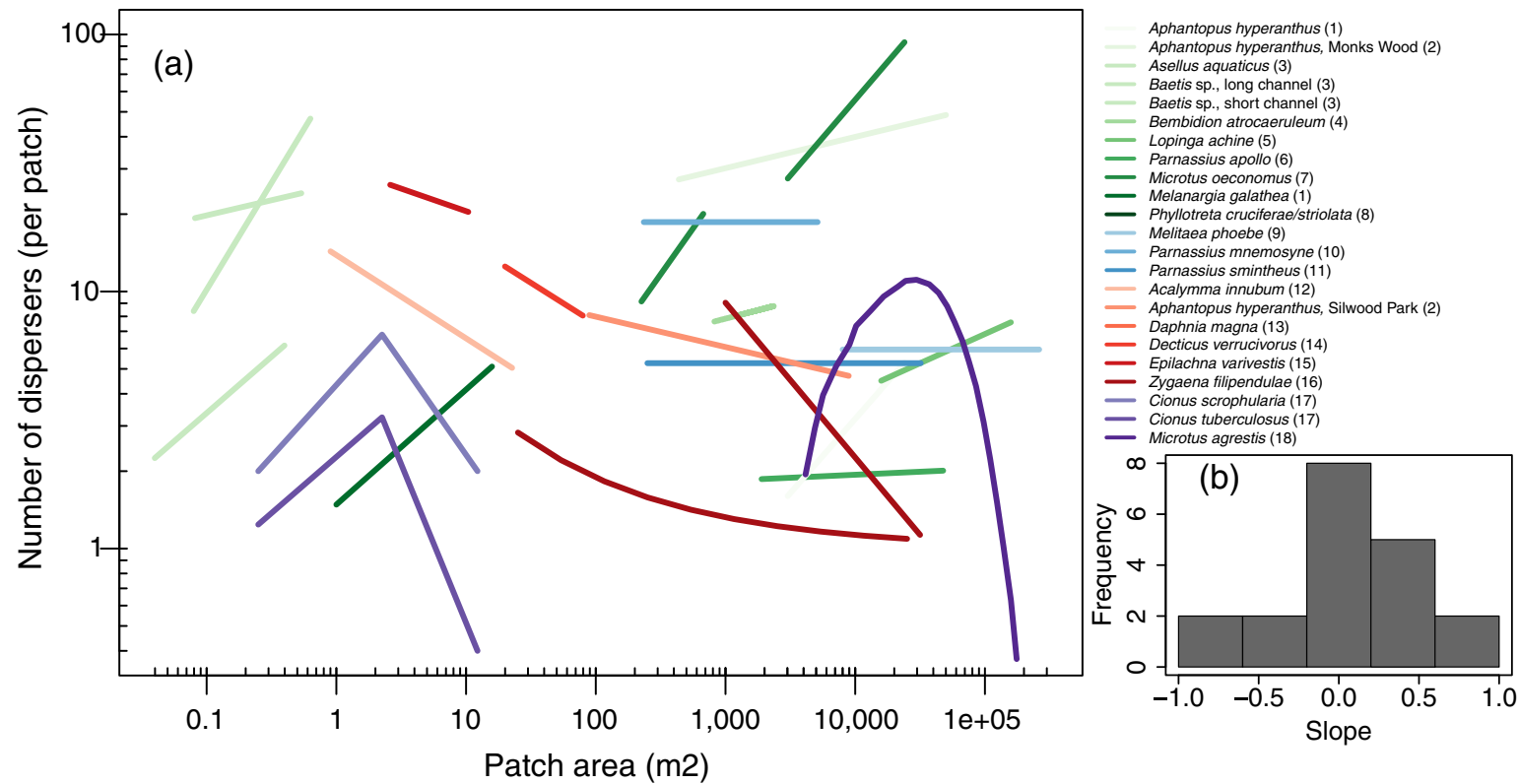

FIG. 2. Different empirically observed patterns of the area dependence of dispersal (ADD). (A) Species-specific predictions between patch area and number of dispersers. Each line is a prediction derived from the original study for the respective species and covers the range of patch sizes assessed. The color code denotes relationships that were positive (green colors), neutral (blue colors), negative (red colors), or hump-shaped (purple colors). The species and respective studies considered are named and numbered, respectively, in the legend: Baguette et al. (2000) (1), Sutcliffe et al. (1997) (2), Englund and Hambäck (2004a,b)(3), Bates et al. (2006) (4), Bergman and Landin (2001) (5), Fred and Brommer (2009) (6), Andreassen and Ims (2001) (7), Kareiva (1985) (8), Wang et al. (2004) (9), Välimäki and Itämies (2003) (10), Roland et al. (2000) (11), Batch (1984) (12), Altermatt and Ebert (2008) (13, here the predictor is patch volume in L), Hjermann (2000) (14), Turchin (1986) (15), Menéndez et al. (2002) (16), Andersson and Hambäck (2012) (17), and Crone et al. (2001) (18). (B) Histogram of the slope estimates of all linear models predicting the relationship between patch area and dispersal (i.e., number of dispersers; estimated on a log-log scale). 
(a)

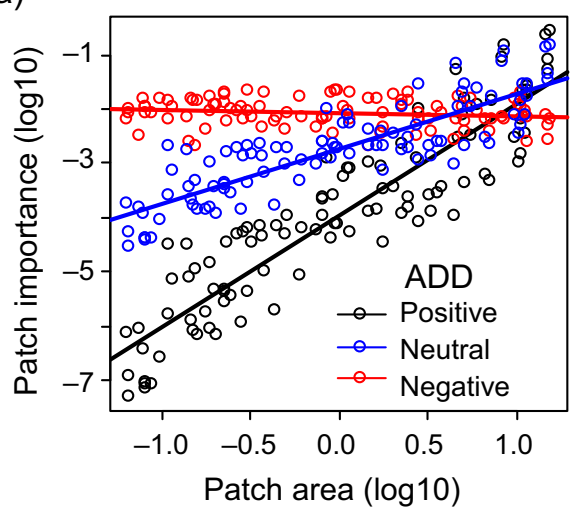

(b)

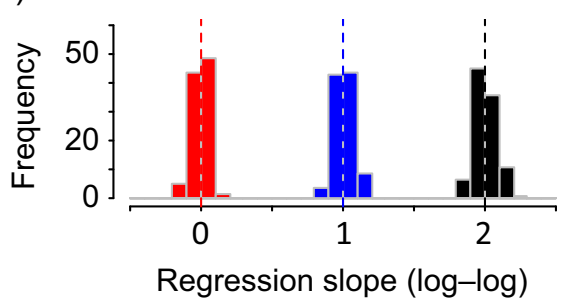

FIG. 3. Relationship between patch importance and area. (a) Scatter plot of patch importance and area from one simulated metapopulations with 100 local patches. Each point shows the area and importance of one patch under the corresponding scenario of ADD. Lines are regression lines. (b) Distribution of regression slopes between patch importance and area on a log$\log$ scale, based on 100 simulated metapopulations. Each metapopulation consists of 100 local patches, with patch areas sampled from a $\log$-uniform distribution, i.e., $\log 2(\mathrm{~A}) \sim U[-4$, $4]$, and locations uniformly distributed within $[0,10] \times[0,10]$. Different colors represent different ADD scenarios, and the dashed lines correspond to theoretical expectations, that is, 2,1 , and 0 (i.e., predictions by Eq. 3). Other parameters: $\alpha=0.5$, $\mathrm{c}=\mathrm{e}=1$.

patches when $\omega>0$, increases with it when $\omega<0$, and has no trend when $\omega>=0$ (Fig. 4a). The $\log -\log$ slopes between $\lambda$ and the number of patches are globally consistent with the theoretical expectations from the neutral cases (Ovaskainen 2003; see Fig. 4c). However, as $\omega$ goes close to 1 (i.e., positive ADD), the deviation between numerical and theoretical results increases (Fig. 4c). Given the total area and number of patches, $\lambda$ increases with the standard deviation of patch area when $\omega>0$ and decreases with it when $-1<\omega>0$ (Fig. 4b,\&thinsp; d). Under the scenarios of neutral $(\omega=0)$ and negative $(\omega=-1)$ ADD, the metapopulation capacity does not change with the standard deviation.

Different scenarios of ADD result in different patterns of metapopulation capacity following habitat destruction (Fig. 5). Consistent with our analytical predictions, habitat deterioration does not alter metapopulation capacity under the negative ADD scenario $(\omega=-1)$, but decrease it in other scenarios (i.e., $\omega=0$ or 1 ), regardless of the order of habitat destruction (Fig. 5). Moreover, if all patches deteriorate in a coherent manner (i.e., proportional decreases for all patches), theory also predicts a linear decrease of metapopulation capacity with habitat area under the neutral ADD scenario $(\omega=0)$ and a quadratical decrease under the positive ADD scenario $(\omega=1$; Fig. $5 a)$. When habitat deterioration happens mostly in large patches, the decrease of metapopulation capacity with area reduction follows similar patterns as the above theoretical predictions (Fig. 5a). When deterioration occurs mostly in small patches, the metapopulation capacity (when $\omega \geq 0$ ) decreases linearly under the neutral ADD scenario, and it decreases first slowly and then fast under the positive ADD scenario (Fig. 5b). When deterioration occurs randomly, the decrease of metapopulation capacity is always linear, regardless of ADD scenarios (Fig. 5c).

The loss of habitat patches reduces metapopulation capacity under all scenarios of ADD and all regimes of habitat loss (Fig. 5d-f). Under the positive and neutral scenarios of $\operatorname{ADD}(\omega=1$ and 0$)$, the metapopulation capacity decreases sharply if large patches are lost first, linearly if patches are lost randomly, but it remains relatively constant if small patches are lost first. Under the negative scenario of $\operatorname{ADD}(\omega=-1)$, metapopulation capacity declines linearly with the number of patches lost, regardless of the order of patch loss.

\section{Discussion}

In this paper, we used a spatially realistic metapopulation model to demonstrate how assumptions on ADD significantly affect metapopulation dynamics and change conclusions about the importance of habitat patches and the consequences of habitat deteriorations, which are of high relevance for conservation biology. Classic metapopulation models have mostly considered positive ADD (Hanski and Ovaskainen 2000, Ovaskainen 2002, 2003, Ovaskainen and Hanski 2003), which contrasts with some empirical findings showing that in some natural metapopulations small patches can produce more dispersers than large ones (Fig. 2). In such systems, our analyses showed that several important and conservation-relevant aspects, especially with respect to metapopulation capacity, could be reversed depending on scenarios of habitat deterioration or habitat loss (Fig. 5).

\section{The area dependence of dispersal}

Our literature review reveals that across different systems, dispersal can either increase or decrease with patch area. Although the occurrence of a negative ADD is not restricted to specific organisms nor to specific environments, we notice particular evidence for this type of metapopulation dynamics from ephemeral habitats, that is, habitats that have a high turnover rate and where patch persistency is often short, and extrinsically driven 
(a)

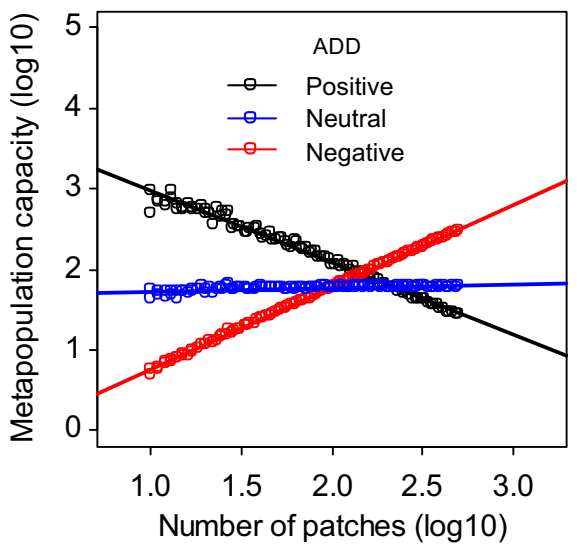

(c)

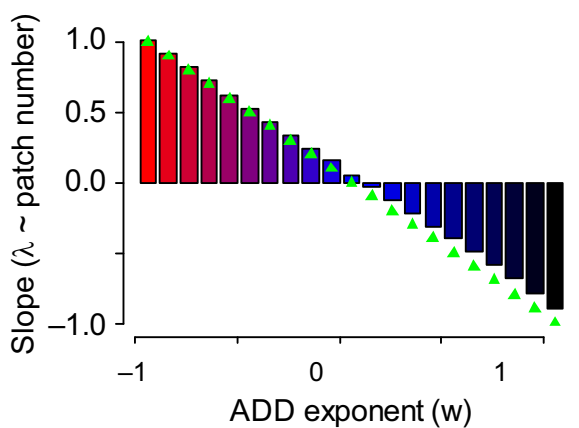

(b)

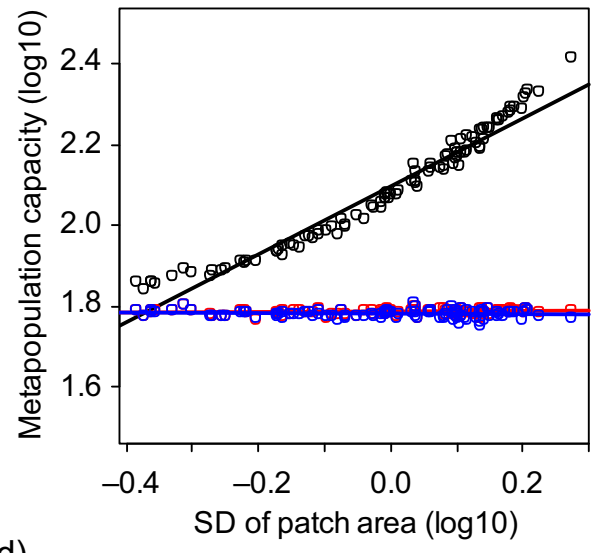

(d)

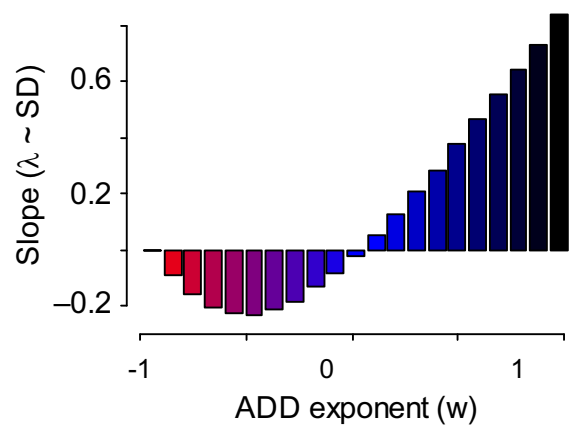

FIG. 4. The dependence of metapopulation capacity on the (a), (c) number and (b), (d) standard deviation of patches, given the total area of patches. For each scenario of ADD, we simulated 100 metapopulations with fixed total area of 100. In (a) and (c), each metapopulation consists of $10-1,000$ patches, with patch areas initially sampled from a $\log -$ uniform distribution (i.e., $\log 2(\mathrm{~A}) \sim U$ $[-4,4])$ and then rescaled proportionally to match the total area. In (b) and (d), each metapopulation consists of 100 patches, with patch areas sampled from log-uniform distributions ranging from $U[-1,1]$ to $U[-5,5]$. In (a) and (b), each point corresponds to one metapopulation, and lines represents fits from simple regressions. Different colors represent the three scenarios of ADD in Fig. 1. In (c) and (d), each bar represents the regression slope between metapopulation capacity and the number of patches (c) or the standard deviation (d) under respective value of ADD exponent. Note that the three scenarios in (a) and (b) are included in (c) and (d). The green triangles in (c) represent the theoretical expectation derived under a homogeneous case. All other parameters are the same as in Fig. 3.

(Reigada et al. 2015, O’Neill 2016). In such habitats, temporal or spatial dispersal is a common response to escape the local patch deterioration (O'Neill 2016, Mahaut et al. 2017), and for these negative ADDs to emerge, we expect different dispersal strategies depending on patch sizes because populations in patches of different sizes experience different levels of stress. Well-known cases of such ephemeral habitats are meadow habitat patches inhabited by butterfly species, and rock pools, tidal pools, vernal pools, or possibly also pitcher-plant communities and the diverse and well-studied invertebrate communities found in those. This includes cladocerans, copepods, or ostracods, which are not only commonly found in such habitats, but which are also characterized by rapid population and colonization dynamics (Srivastava et al. 2004, Altermatt et al. 2007, 2009, 2012, Altermatt and Ebert 2008, Vanschoenwinkel et al. 2008). Importantly, several of these organisms possess drought-resistant resting stages that are also their dispersal propagule. Thus, their strategy to persist during desiccation events (one of the drivers of pond ephemerality) covers both local persistence and dispersal (Altermatt and Ebert 2008, 2010, Vanschoenwinkel et al. 2008, Altermatt et al. 2009). This may also be a more general criterion to look for negative ADD: we expect that it is likely to occur in cases where the evolutionary/ecological strategy to persist unfavorable conditions (such as a drought in a pond) is the same strategy/life stage central to dispersal. In such a scenario, and more generally stated, when the rate of externally driven extinctions is inversely related to patch area, selection may favor a strong nonlinear decrease of dispersal with increasing area (or an increase with increasing succession status; Ronce et al. 2005). Importantly, however, for a negative ADD to emerge, such a decrease in dispersal propensity with increasing patch area must 


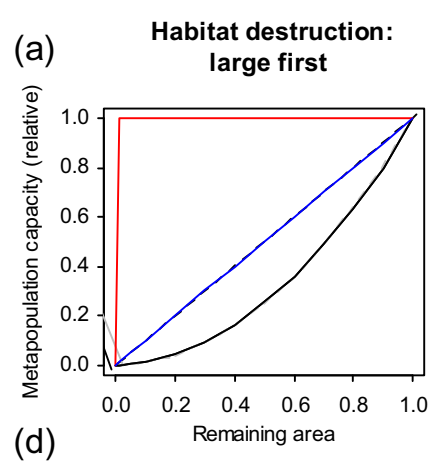

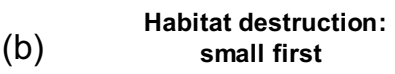

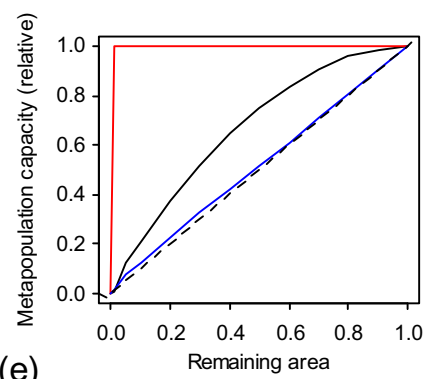

(e)

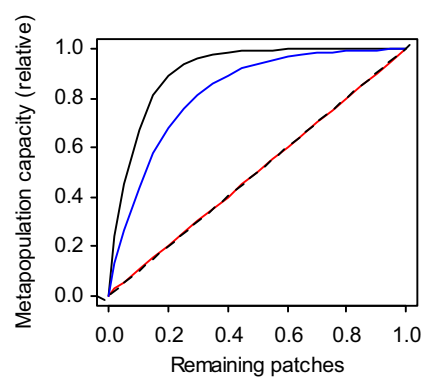

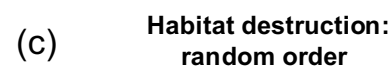

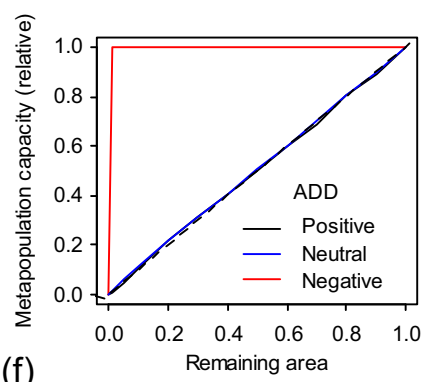

Habitat deterioration

f)

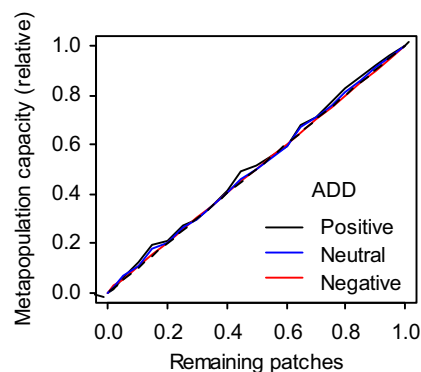

Habitat loss

FIG. 5. Change of metapopulation capacity with (a)-(c) habitat deterioration and (d)-(f) loss, following three regimes: (a), (d) large patches first, (b), (e) small patches first, and (c), (f) and random order. Under each scenario of ADD and regime of habitat destruction, we simulated the gradual deterioration and loss of patches across 100 metapopulations, with initial parameters as in Fig. 3. In the simulation of habitat deterioration (a)-(c), we first rank the patches following the three regimes and then continuously reduce the area of patches (no smaller than 0.01) to match the predefined degree of deterioration. For habitat loss (d)-(f), we remove patches sequentially following the three regimes. Each curve represents the median of relative metapopulation capacity as a function of the remaining relative area (a)-(c) or number (d)-(f) of patches. In (a), the blue line and black curve overlap with the 1:1 line and the quadratic curve, respectively.

be sufficiently steep to compensate for the increase in dispersal resulting from larger population sizes observed in larger patches.

The relevance of ADD may increase with ongoing climate change: Many ecosystems are expected to change in their state, and mechanisms associated with ADD, such as dispersal or demography, are predicted to be especially vulnerable to climate change (Urban et al. 2016). We thus speculate that the study of ADD would be especially relevant when matched with the study of dispersal strategies, dispersal syndromes, and dispersal genetics (Cote et al. 2016, Fronhofer et al. 2018, Saastamoinen et al. 2018). A further possible interesting link between ADD and existing ecological literature is with respect to nonlinear dispersal responses and recruitment, such as tests of the Janzen-Connell model (Terborgh et al. 2008) or inverse density-dependent dispersal (Little et al. 2019). These are related examples and scenarios of ecological dynamics that follow inverse relationships, for example, that recruitment of plant saplings is scarce near reproductive conspecifics, even though seed set may be highest. The explanation is that negative effects of predators or pathogens is highest at high population densities, and that this effect decreases with increasing distance. Thus, recruitment is higher in populations that are small and or with scattered individuals, and low in large and dense populations. We conclude that negative (inverse) relationships between dispersal and demographic or environmental properties may be more abundant than generally assumed.

Our empirical results (Fig. 2) are not based on a formal and quantitative meta-analysis, but rather give a qualitative reasoning for all possible ADD to be found in natural systems. Generally, to test our model assumptions, one needs to collect data on the number of total emigrants and patch area, rather than dispersal propensity and population density only. While doing our literature search, we found many studies that likely collected such information on total number of dispersers, but then transformed it into dispersal rates, and we could not use these values for a direct comparison with our model. Thus, we highlight the importance of recording and reporting raw data on both patch area and absolute number of dispersers per patch. Such a practice will allow an easier and much better integration of empirical findings and the respective metapopulation models.

\section{Conservation implications of $A D D$}

Are larger or smaller patches more important in maintaining the persistence of metapopulations? The answer differs substantially among different ADD scenarios. As our model clarifies, the contribution of each patch to metapopulation persistence is jointly determined by the 
ADD and extinction rates (i.e., Eq. 2). Under the classic assumption that dispersal increases linearly and extinction likelihoods decreases inversely with area, a larger patch has higher contribution that scales quadratically with its area (see also Hanski and Ovaskainen 2000). In contrast, under the scenario that dispersal and extinction both decrease inversely with area, every patch has roughly the same contribution to the persistence of the metapopulation. This counterintuitive result, which is explained by the trade-off between dispersal and extinction across patches with different areas, has direct implications for conservation in this type of ecosystems.

A long-standing debate in conservation biology is whether we should have a single large (SL) or several small (SS) reserves (the so-called SLOSS problem, Diamond 1975, Simberloff and Abele 1976, Ovaskainen 2002, McCarthy et al. 2011). Our results demonstrate that the answer to this question also depends on the scenario of ADD. Under the classic assumption of a positive ADD, a larger patch contributes more than proportional to the MC, and hence landscapes with a few larger patches (i.e., SL) can better support metapopulations than those with many smaller patches. Thus, SL is better than SS (Ovaskainen 2002). However, under the scenario of a neutral ADD, landscapes with a few large patches and many small patches have roughly same MC, because the contribution of a patch to MC increases proportionally with its area. Thus, SL is similar as SS. Under a negative ADD, all patches have similar contribution regardless of their areas, so landscapes with more smaller patches can better support metapopulations than those with a few larger patches. Thus, SS is better than SL. Although early studies derived similar conclusions under a homogeneous case (Ovaskainen 2002), our analyses extend this to more realistic cases of heterogeneous patch size distributions.

To devise a better conservation strategy given total habitat area, a related question is whether we should maintain more or less evenly distributed patch sizes? Our results show that ADD assumptions also alter answers to this question. Under a positive ADD, a higher variation in patch size distribution increases the metapopulation capacity. This is because a higher variance in patch size distribution implies a higher probability of larger patches, which contributed more than proportionally to metapopulation capacity. Under a negative ADD, a more even patch size distribution is beneficial to the persistence of metapopulations.

Therefore, the assumption of ADD alters our predictions on the importance of individual patches and the configuration of patch networks. As a consequence, it also alters the response of metapopulation capacity to habitat destruction. Habitat deterioration, which reduces the area but not the number of patches, decreases metapopulation capacity under the positive and neutral ADD scenarios, but has no effect under the negative ADD scenario (Fig. 5a). Habitat loss, which reduces both the total area and number of patches, decreases the metapopulation capacity under all scenarios of ADD. However, different regimes of habitat loss generate different patterns of metapopulation capacity under different assumptions of ADD.

\section{Conclusion}

Our study shows that an effective management strategy for metapopulations is context dependent of ADD. If dispersal is positively correlated with patch area, efforts should be devoted to preserving large patches and the total habitat area. If dispersal is negatively correlated with patch area, the most efficient strategy is to preserve a high number of patches, even small ones. Our results highlight the importance of detailed knowledge about the ADD in the focal spatial system for planning conservation strategies. Heuristically, our study demonstrates in principle that efficient management may often require context-dependent strategies considering the details of the target systems. Therefore, future studies incorporating system-specific knowledge into models are key to achieve a more practical theory for landscape management.

\section{ACKNOWLEDGMents}

Both authors contributed equally to the manuscript. We thank Isabelle Gounand, Felix Moerman, two reviewers, and the editor J. M. Ponciano for comments on the manuscript. We thank Göran Englund and Peter Hambäck for raw data on their published studies. Funding is from the Swiss National Science Foundation Grant No PP00P3_150698 and the University of Zurich Research Priority Programme URPP Global Change and Biodiversity (to FA) and from the National Key Research and Development Program of China (2017YFC0503906; to SW).

\section{Literature Cited}

Altermatt, F., and D. Ebert. 2008. The influence of pool volume and summer desiccation on the production of the resting and dispersal stage in a Daphnia metapopulation. Oecologia 157:441-452.

Altermatt, F., and D. Ebert. 2010. Populations in small, ephemeral habitat patches may drive dynamics in a Daphnia magna metapopulation. Ecology 91:2975-2982.

Altermatt, F., J. Hottinger, and D. Ebert. 2007. Parasites promote host gene flow in a metapopulation. Evolutionary Ecology 21:561-575.

Altermatt, F., V. I. Pajunen, and D. Ebert. 2008. Climate change affects colonization dynamics in a metacommunity of three Daphnia species. Global Change Biology 14:1209-1220.

Altermatt, F., I. Pajunen, and D. Ebert. 2009. Desiccation of rock pool habitats and its influence on population persistence in a Daphnia metacommunity. PLoS ONE 4:e4703.

Altermatt, F., A. Bieger, and S. Morgan. 2012. Habitat characteristics and metapopulation dynamics of the copepod Tigriopus californicus. Marine Ecology Progress Series 468:85-93.

Andersson, P., and P. A. Hambäck. 2012. What shapes local density? The importance of migration rates and local growth for density-patch size relationships in two Cionus weevils. Ecological Entomology 37:90-98.

Andreassen, H. P., and R. A. Ims. 2001. Dispersal in patchy vole populations: role of patch configuration, density dependence, and demography. Ecology 82:2911-2926. 
Baguette, M., S. Petit, and F. Quéva. 2000. Population spatia structure and migration of three butterfly species within the same habitat network: consequences for conservation. Journal of Applied Ecology 37:100-108.

Batch, C. E. 1984. Plant spatial pattern and herbivore population dynamics: plant factors affecting the movement patterns of a tropical cucurbit specialist (Acalymma innubum). Ecology 65:175-190.

Bates, A. J., J. P. Sadler, and A. P. Fowles. 2006. Conditiondependent dispersal of a patchily distributed riparian ground beetle in response to disturbance. Oecologia 150:50-60.

Berendonk, T. U., K. Spitze, and W. C. Kerfoot. 2009. Ephemeral metapopulations show high genetic diversity at regional scales. Ecology 90:2670-2675.

Bergman, K.-O., and J. Landin. 2001. Distribution of occupied and vacant sites and migration of Lopinga achine (Nymphalidae: Satyrinae) in a fragmented landscape. Biological Conservation 102:183-190.

Clobert, J., E. Danchin, A. A. Dhondt, and J. D. Nichols, editors. 2001. Dispersal. Oxford University Press, Oxford, UK

Cote, J., E. Bestion, S. Jacob, J. Travis, D. Legrand, and M. Baguette. 2016. Evolution of dispersal strategies and dispersal syndromes in fragmented landscapes. Ecography 40:5673

Crone, E. E., D. Doak, and J. Pokki. 2001. Ecological influences on the dynamics of a field vole metapopulation. Ecology 82:831-843

De Roissart, A., S. Wang, and D. Bonte. 2015. Spatial and spatiotemporal variation in metapopulation structure affects population dynamics in a passively dispersing arthropod. Journal of Animal Ecology 84:1565-1574.

Diamond, J. M. 1975. The island dilemma: lessons of modern biogeographic studies for the design of natural reserves. Biological Conservation 7:129-146.

Englund, G., and P. A. Hambäck. 2004a. Scale dependence of emigration rates. Ecology 85:320-327.

Englund, G., and P. A. Hambäck. 2004b. Scale-dependence of movement rates in stream invertebrates. Oikos 105:31-40.

Fournier, B., N. Mouquet, M. A. Leibold, and D. Gravel. 2017. An integrative framework of coexistence mechanisms in competitive metacommunities. Ecography 40:630-641.

Fred, M. S., and J. E. Brommer. 2009. Resources influence dispersal and population structure in an endangered butterfly Insect Conservation and Diversity 2:176-182.

Fronhofer, E. A., and F. Altermatt. 2017. Classical metapopulation dynamics and eco-evolutionary feedbacks in dendritic networks. Ecography 40:1455-1466.

Fronhofer, E. A., J. Klecka, C. J. Melian, and F. Altermatt. 2015. Condition-dependent movement and dispersal in experimental metacommunities. Ecology Letters 18:954-963.

Fronhofer, E. A. et al. 2018. Bottom-up and top-down control of dispersal across major organismal groups. Nature Ecology and Evolution 2:1859-1863.

Gilarranz, L. J., and J. Bascompte. 2012. Spatial network structure and metapopulation persistence. Journal of Theoretical Biology 297:11-16.

Gilpin, M., and I. Hanski, editors. 1991. Metapopulation dynamics: empirical and theoretical investigations. Academic Press, London, UK.

Gyllenberg, M., and I. Hanski. 1992. Single-species metapopulation dynamics - a structured model. Theoretical Population Biology 42:35-61.

Gyllenberg, M., and I. Hanski. 1997. Habitat deterioration, habitat destruction, and metapopulation persistence in a heterogenous landscape. Theoretical Population Biology 52: $198-215$.
Hambäck, P. A., and G. Englund. 2005. Patch area, population density and the scaling of migration rates: the resource concentration hypothesis revisited. Ecology Letters 8:10571065.

Hanski, I. 1998. Metapopulation dynamics. Nature 396:41-49.

Hanski, I. 1999. Habitat connectivity, habitat continuity, and metapopulations in dynamic landscapes. Oikos 87:209-219.

Hanski, I., and O. E. Gaggiotti, editors. 2004. Ecology, genetics and evolution of metapopulations. Elsevier Academic Press, Amsterdam, Netherlands.

Hanski, I., and M. Gyllenberg. 1993. Two general metapopulation models and the core-satellite species hypothesis. American Naturalist 142:17-41.

Hanski, I., and O. Ovaskainen. 2000. The metapopulation capacity of a fragmented landscape. Nature 404:755-758.

Hanski, I., and I. J. Saccheri. 2006. Molecular-level variation affects population growth in a butterfly metapopulation. PLoS Biology 4:719-726.

Hanski, I., T. Mononen, and O. Ovaskainen. 2011. Eco-evolutionary metapopulation dynamics and the spatial scale of adaptation. The American Naturalist 177:29-43.

Harrison, S. 1991. Local extinction in a metapopulation context: an empirical evaluation. Biological Journal of the Linnean Society 42:73-88.

Hill, J. K., C. D. Thomas, and O. T. Lewis. 1996. Effects of habitat patch size and isolation on dispersal by Hesperia comma butterflies: implications for metapopulation structure. Journal of Animal Ecology 65:725-735.

Hjermann, D. 2000. Spatial ecology of the bush-cricket Decticus verrucivorus: Movement, metapopulation dynamics and genetic variation in patchy and linear habitats. University of Oslo, Oslo, Norway.

Jansen, V. A. A., and R. Vitalis. 2007. The evolution of dispersal in a Levins' type metapopulation. Evolution 61:2386-2397.

Kareiva, P. 1985. Finding and losing host plants by Phyllotreta: patch size and surrounding habitat. Ecology 66:1809-1816.

Kindvall, O. 1999. Dispersal in a metapopulation of the bush cricket, Metrioptera bicolor (Orthoptera: Tettigoniidae). Journal of Animal Ecology 68:172-185.

Kindvall, O., and A. Petersson. 2000. Consequences of modelling interpatch migration as a function of patch geometry when predicting metapopulation extinction risk. Ecological Modelling 129:101-109.

Leibold, M. A., et al. 2004. The metacommunity concept: a framework for multi-scale community ecology. Ecology Letters 7:601-613.

Levin, S. A. 1992. The problem of pattern and scale in ecology. Ecology 73:1943-1967.

Levins, R. 1969. Some demographic and genetic consequences of environmental heterogeneity for biological control. Bulletin of the Entomological Society of America 15:237-240.

Levins, R. 1970. Extinction. Pages 77-107 in M. Gerstenhaber, editor. Some mathematical problems in biology. The American Mathematical Society, Providence, Rhode Isalnd, USA.

Little, C. J., A. Fronhofer Emanuel, and F. Altermatt. 2019. Nonlinear effects of intraspecific competition alter landscapewide upscaling of ecosystem function. bioRxiv. https://doi. org/10.1101/470591

Mahaut, L., G. Fried, and S. Gaba. 2017. Patch dynamics and temporal dispersal partly shape annual plant communities in ephemeral habitat patches. Oikos 127:147-159.

Mari, L., R. Casagrandi, E. Bertuzzo, A. Rinaldo, and M. Gatto. 2014. Metapopulation persistence and species spread in river networks. Ecology Letters 14:426-434.

Matthysen, E. 2005. Density-dependent dispersal in birds and mammals. Ecography 28:403-416. 
McCarthy, M. A., C. J. Thompson, A. L. Moore, and H. P. Possingham. 2011. Designing nature reserves in the face of uncertainty. Ecology Letters 14:470-475.

Menéndez, R., D. Gutiérrez, and C. D. Thomas. 2002. Migration and Allee effects in the six-spot burnet moth Zygaena filipendulae. Ecological Entomology 27:317-325.

Metz, J. A., and M. Gyllenberg. 2001. How should we define fitness in structured metapopulation models? Including an application to the calculation of evolutionarily stable dispersal strategies. Proceedings of the Royal Society B 268:499-508.

Olivieri, I., Y. Michalakis, and P. H. Gouyon. 1995. Metapopulation genetics and the evolution of dispersal. American Naturalist 146:202-228.

O'Neill, B. J. 2016. Community disassembly in ephemeral ecosystems. Ecology 97:3285-3292.

Ovaskainen, O. 2002. Long-term persistence of species and the SLOSS problem. Journal of Theoretical Biology 218:419-433.

Ovaskainen, O. 2003. Habitat destruction, habitat restoration and eigenvector-eigenvalue relations. Mathematical Biosciences 181:165-176.

Ovaskainen, O., and I. Hanski. 2001. Spatially structured metapopulation models: global and local assessment of metapopulation capacity. Theoretical Population Biology 60:281-302

Ovaskainen, O., and I. Hanski. 2003. How much does an individual habitat fragment contribute to metapopulation dynamics and persistence? Theoretical Population Biology 64:481-495.

Poethke, H. J., and T. Hovestadt. 2002. Evolution of densityand patch-size-dependent dispersal rates. Proceedings of the Royal SocietySeries B 269:637.

Pokki, J. 1981. Distribution, demography and dispersal of the field vole, Microtus agrestis (L.), in the Tvärminne Archipelago, Finland. Acta Zoologica Fennica 164:1-48.

Purves, D. W., and J. Dushoff. 2005. Directed seed dispersal and metapopulation response to habitat loss and disturbance: application to Eichhornia paniculata. Journal of Ecology 93:658-669.

Reigada, C., S. Schreiber, F. Altermatt, and M. Holyoak. 2015. Metapopulation dynamics on ephemeral patches. American Naturalist 185:183-195.

Roland, J., N. Keyghobadi, and S. Fownes. 2000. Alpine Parnassius butterfly dispersal: effects of landscape and population size. Ecology 81:1642-1653.

Ronce, O. 2007. How does it feel to be like a rolling stone? Ten questions about dispersal evolution. Annual Review of Ecology, Evolution, and Systematics 38:231-253.

Ronce, O., S. Brachet, I. Olivieri, P.-H. Gouyon, and J. Clobert. 2005. Plastic changes in seed dispersal along ecological succession: theoretical predictions from an evolutionary model. Journal of Ecology 93:431-440.

Saastamoinen, M. et al. 2018. Genetics of dispersal. Biological Reviews 93:574-599.
Schtickzelle, N., and M. Baguette. 2003. Behavioural responses to habitat patch boundaries restrict dispersal and generate emigration-patch area relationships in fragmented landscapes. Journal of Animal Ecology 72:533-545.

Simberloff, D. S., and L. G. Abele. 1976. Island biogeography theory and conservation practice. Science 191:285-286.

Srivastava, D. S., J. Kolasa, J. Bengtsson, A. Gonzalez, S. P. Lawler, T. E. Miller, P. Munguia, T. Romanuk, D. C. Schneider, and M. K. Trzcinski. 2004. Are natural microcosms useful model systems for ecology? Trends in Ecology and Evolution 19:379-384.

Stamps, J. A., M. Buechner, and V. V. Krishnan. 1987. The effects of edge permeability and habitat geometry on emigration from patches of habitat. American Naturalist 129:533-552.

Støen, O.-G., A. Zedrosser, S. Sæbø, and J. E. Swenson. 2006. Inversely density-dependent natal dispersal in brown bears Ursus arctos. Oecologia 148:356.

Sutcliffe, O. L., C. D. Thomas, and D. Peggie. 1997. Areadependent migration by Ringlet butterflies generates a mixture of patchy population and metapopulation attributes. Oecologia 109:229-234.

Terborgh, J., G. Nuñez-Iturri, N. C. A. Pitman, F. H. C. Valverde, P. Alvarez, V. Swamy, E. G. Pringle, and C. E. T. Paine. 2008. Tree recruitment in an empty forest. Ecology 89:17571768 .

Travis, J. M. J., and C. Dytham. 1998. The evolution of dispersal in a metapopulation: a spatially explicit, individual-based model. Proceedings of the Royal Society B 265:17-23.

Travis, J. M. J., D. J. Murrell, and C. Dytham. 1999. The evolution of density-dependent dispersal. Proceedings of the Royal Society B 266:1837-1842.

Turchin, P. B. 1986. Modelling the effect of host patch size on Mexican bean beetle emigration. Ecology 67:124-132.

Urban, M. C. et al. 2016. Improving the forecast for biodiversity under climate change. Science 353:aad8466.

Välimäki, P., and J. Itämies. 2003. Migration of the clouded apollo butterfly Parnassius mnemosyne in a network of suitable habitats: effects of patch characteristics. Ecography 26:679-691.

Vanschoenwinkel, B., S. Gielen, M. Seaman, and L. Brendonck. 2008. Any way the wind blows -frequent wind dispersal drives species sorting in ephemeral aquatic communities. Oikos 117:125-134.

Wahlberg, N., T. Klemetti, V. Selonen, and I. Hanski. 2002. Metapopulation structure and movements in five species of checkerspot butterflies. Oecologia 130:33-43.

Wang, R., Y. Wang, J. Chen, G. Lei, and R. Xu. 2004. Contrasting movement patterns in two species of chequerspot butterflies, Euphydryas aurinia and Melitaea phoebe, in the same patch network. Ecological Entomology 29:367-374.

Wang, S., B. Haegeman, and M. Loreau. 2015. Dispersal and metapopulation stability. PeerJ 3:e1295. 